UDC: $517.518 .28: 517.983 .2$

45 (LXXI) No.1 (2021), 71-83

DOI: $10.37560 /$ matbil21451071d

\title{
SEVERAL INEQUALITIES FOR OPERATOR MONOTONE FUNCTIONS ON FINITE INTERVALS
}

\author{
SILVESTRU SEVER DRAGOMIR ${ }^{1,2}$
}

\begin{abstract}
In this paper we show that, if the function $f:(-1,1) \rightarrow \mathbb{R}$ is operator monotone in $(-1,1)$, then there exists a positive measure $\mu$ on $[-1,1]$ such that

$$
\begin{aligned}
& {[f(B)-f(A)](B-A)} \\
& =f^{\prime}(0) \int_{-1}^{1}\left(\int_{0}^{1}\left[(1-\lambda((1-t) A+t B))^{-1}(B-A)\right]^{2} d t\right) d \mu(\lambda)
\end{aligned}
$$

for all $A, B$ with $\mathrm{S}_{\mathrm{p}}(A), \mathrm{S}_{\mathrm{p}}(B) \subset(-1,1)$. Some necessary and sufficient conditions for the operators $A, B$ with $\operatorname{Sp}(A), \operatorname{Sp}(B) \subset(-1,1)$ such that the inequality

$$
f(B) B+f(A) A \geq f(A) B+f(B) A
$$

holds for any operator monotone function $f$ on $(-1,1)$ are also given.
\end{abstract}

\section{INTRODUCTION}

Consider a complex Hilbert space $(H,\langle\cdot, \cdot\rangle)$. An operator $T$ is said to be positive (denoted by $T \geq 0$ ) if $\langle T x, x\rangle \geq 0$ for all $x \in H$ and also an operator $T$ is said to be strictly positive (denoted by $T>0$ ) if $T$ is positive and invertible. A real valued continuous function $f(t)$ on $(0, \infty)$ is said to be operator monotone if $f(A) \geq f(B)$ holds for any $A \geq B>0$.

In 1934, K. Löwner [10 had given a definitive characterization of operator monotone functions as follows, see for instance [2, p. 144-145]:

Theorem 1. A function $f:[0, \infty) \rightarrow \mathbb{R}$ is operator monotone in $[0, \infty)$ if and only if it has the representation

$$
f(t)=a+b t+\int_{0}^{\infty} \frac{t s}{t+s} d m(s)
$$

2010 Mathematics Subject Classification. 47A63, 26D15, 26D10.

Key words and phrases. Operator monotone functions, Integral inequalities, Operator inequality. 
where $a \in \mathbb{R}$ and $b \geq 0$ and a positive measure $m$ on $(0, \infty)$ such that

$$
\int_{0}^{\infty} \frac{s}{1+s} d m(s)<\infty .
$$

We recall the important fact proved by Löwner and Heinz that states that the power function $f:(0, \infty) \rightarrow \mathbb{R}, f(t)=t^{\alpha}$ is an operator monotone function for any $\alpha \in[0,1], 8$.

In [6], T. Furuta observed that for $\alpha_{j} \in[0,1], j=1, \ldots, n$ the functions

$$
g(t):=\left(\sum_{j=1}^{n} t^{-\alpha_{j}}\right)^{-1} \text { and } h(t)=\sum_{j=1}^{n}\left(1+t^{-1}\right)^{-\alpha_{j}}
$$

are operator monotone in $(0, \infty)$.

Let $f(t)$ be a continuous function $(0, \infty) \rightarrow(0, \infty)$. It is known that $f(t)$ is operator monotone if and only if $g(t)=t / f(t)=: f^{*}(t)$ is also operator monotone, see for instance [6] or [11.

Consider the family of functions defined on $(0, \infty)$ and $p \in[-1,2] \backslash\{0,1\}$ by

$$
f_{p}(t):=\frac{p-1}{p}\left(\frac{t^{p}-1}{t^{p-1}-1}\right)
$$

and

$$
f_{0}(t):=\frac{t}{1-t} \ln t, f_{1}(t):=\frac{t-1}{\ln t} \text { (logarithmic mean). }
$$

We also have the functions of interest

$$
\left.f_{-1}(t)=\frac{2 t}{1+t}(\text { harmonic mean }), f_{1 / 2}(t)=\sqrt{t} \text { (geometric mean }\right) .
$$

In [5] the authors showed that $f_{p}$ is operator monotone for $1 \leq p \leq 2$.

In the same category, we observe that the function

$$
g_{p}(t):=\frac{t-1}{t^{p}-1}
$$

is an operator monotone function for $p \in(0,1]$, [7].

It is well known that the logarithmic function $\ln$ is operator monotone and in 6. the author obtained that the functions

$$
f(t)=t(1+t) \ln \left(1+\frac{1}{t}\right), g(t)=\frac{1}{(1+t) \ln \left(1+\frac{1}{t}\right)}
$$

are also operator monotone functions on $(0, \infty)$.

The case of operator monotone functions on finite intervals is as follows [2, $\mathrm{p}$. 134]:

Theorem 2. Let $f$ be a nonconstant operator monotone function on $(-1,1)$. Then there exists a unique probability measure $\mu$ on $[-1,1]$ such that

$$
f(t)=f(0)+f^{\prime}(0) \int_{-1}^{1} \frac{t}{1-\lambda t} d \mu(\lambda)
$$

for $t \in(-1,1)$, where $f^{\prime}(0)>0$. 
It is well known that the function $f:\left(-\frac{\pi}{2}, \frac{\pi}{2}\right) \rightarrow \mathbb{R}, f(t)=\tan t$ is operator monotone on $\left(-\frac{\pi}{2}, \frac{\pi}{2}\right)$ and the function $g$ is operator monotone on $(0, \infty)$ if and only if the function $f:(-1,1) \rightarrow \mathbb{R}, f(t):=g\left(\frac{1+t}{1-t}\right)$ is operator monotone on $(-1,1)$, [13]. Therefore, the functions $f(t)=\left(\frac{1+t}{1-t}\right)^{r}$ and $f(t)=\ln \left(\frac{1+t}{1-t}\right)$ are operator monotone on $(-1,1)$.

For recent operator inequalities related to operator monotone functions, see [1], [3], [9, [12] and [14].

In this paper we show that, if the function $f:(-1,1) \rightarrow \mathbb{R}$ is operator monotone in $(-1,1)$, then there exists a positive measure $\mu$ on $[-1,1]$ such that

$$
\begin{aligned}
& {[f(B)-f(A)](B-A)} \\
& =f^{\prime}(0) \int_{-1}^{1}\left(\int_{0}^{1}\left[(1-\lambda((1-t) A+t B))^{-1}(B-A)\right]^{2} d t\right) d \mu(\lambda)
\end{aligned}
$$

for all $A, B$ with $\operatorname{Sp}(A), \operatorname{Sp}(B) \subset(-1,1)$. Some necessary and sufficient conditions for the operators $A, B$ with $\operatorname{Sp}(A), \operatorname{Sp}(B) \subset(-1,1)$ such that the inequality

$$
f(B) B+f(A) A \geq f(A) B+f(B) A
$$

holds for any operator monotone function $f$ on $(-1,1)$ are also given.

\section{Main Results}

We have the following representation results that is of interest in itself as well:

Theorem 3. Let $f$ be a nonconstant operator monotone function on $(-1,1)$ that satisfies the representation (1.2). Then,

$$
\begin{aligned}
f(B)-f(A) & =f^{\prime}(0) \int_{-1}^{1}\left(\int_{0}^{1}(1-\lambda((1-t) A+t B))^{-1}\right. \\
& \left.\times(B-A)(1-\lambda((1-t) A+t B))^{-1} d t\right) d \mu(\lambda)
\end{aligned}
$$

for all $A, B$ with $\operatorname{Sp}(A), \operatorname{Sp}(B) \subset(-1,1)$.

Proof. For $C$ a selfadjoint operator with $\operatorname{Sp}(C) \subset(-1,1)$, then by 1.2 we have

$$
f(C)=f(0)+f^{\prime}(0) \int_{-1}^{1} C(1-\lambda C)^{-1} d \mu(\lambda) .
$$

Therefore, for $A, B$ with $\operatorname{Sp}(A)$, mathrmSp $(B) \subset(-1,1)$,

$$
f(B)-f(A)=f^{\prime}(0) \int_{-1}^{1}\left[B(1-\lambda B)^{-1}-A(1-\lambda A)^{-1}\right] d \mu(\lambda) .
$$

For $\lambda \in[-1,1], \lambda \neq 0$ we put

$$
U_{\lambda}:=B(1-\lambda B)^{-1}-A(1-\lambda A)^{-1} .
$$


Then

$$
\begin{aligned}
\lambda U_{\lambda} & =\lambda B(1-\lambda B)^{-1}-\lambda A(1-\lambda A)^{-1} \\
& =(1-1+\lambda B)(1-\lambda B)^{-1}-(1-1+\lambda A)(1-\lambda A)^{-1} \\
& =(1-\lambda B)^{-1}-1-\left((1-\lambda A)^{-1}-1\right) \\
& =(1-\lambda B)^{-1}-(1-\lambda A)^{-1}
\end{aligned}
$$

namely

$$
U_{\lambda}=\frac{(1-\lambda B)^{-1}-(1-\lambda A)^{-1}}{\lambda}
$$

for $\lambda \in[-1,1], \lambda \neq 0$.

Using the identity

$$
X^{-1}-Y^{-1}=X^{-1}(Y-X) Y^{-1}
$$

we get

$$
\begin{aligned}
& (1-\lambda B)^{-1}-(1-\lambda A)^{-1} \\
& =(1-\lambda B)^{-1}(1-\lambda A-1+\lambda B)(1-\lambda A)^{-1} \\
& =\lambda(1-\lambda B)^{-1}(B-A)(1-\lambda A)^{-1}
\end{aligned}
$$

and then

$$
\lambda U_{\lambda}=\lambda(1-\lambda B)^{-1}(B-A)(1-\lambda A)^{-1}
$$

for $\lambda \in[-1,1], \lambda \neq 0$.

Therefore

$$
U_{\lambda}=(1-\lambda B)^{-1}(B-A)(1-\lambda A)^{-1}, \lambda \in[-1,1]
$$

and we get the identity of interest

$$
f(B)-f(A)=f^{\prime}(0) \int_{-1}^{1}(1-\lambda B)^{-1}(B-A)(1-\lambda A)^{-1} d \mu(\lambda) .
$$

For small $t$ around 0 we have

$$
\begin{aligned}
& f(A+t C)-f(A) \\
& =f^{\prime}(0) t \int_{-1}^{1}\left[(1-\lambda(A+t C))^{-1} C(1-\lambda A)^{-1}\right] d \mu(\lambda),
\end{aligned}
$$

for $C \in B(H), C$ selfadjoint and $A$ with $\operatorname{Sp}(A) \subset(-1,1)$, which implies that the Gâteaux derivative in $A$ of $f$ exists and

$$
\nabla_{A} f(C)=f^{\prime}(0) \int_{-1}^{1}(1-\lambda A)^{-1} C(1-\lambda A)^{-1} d \mu(\lambda) .
$$

Let $A, B$ with $\operatorname{Sp}(A), \operatorname{Sp}(B) \subset(-1,1)$. Consider the auxiliary function $\varphi_{A, B}$ : $[0,1] \rightarrow B(H)$

Then

$$
\varphi_{A, B}(t):=f((1-t) A+t B) .
$$

$$
\frac{d \varphi_{A, B}(t)}{d t}=\nabla_{(1-t) A+t B} f(B-A), t \in(-1,1)
$$


and by 2.3 we get

$$
\begin{aligned}
\frac{d \varphi_{A, B}(t)}{d t} & =f^{\prime}(0) \int_{-1}^{1}(1-\lambda((1-t) A+t B))^{-1} \\
& \times(B-A)(1-\lambda((1-t) A+t B))^{-1} d \mu(\lambda)
\end{aligned}
$$

for $t \in(-1,1)$.

Since

$$
f(B)-f(A)=\varphi_{A, B}(1)-\varphi_{A, B}(0)=\int_{0}^{1} \frac{d \varphi_{A, B}(t)}{d t} d t,
$$

hence by 2.4 we get

$$
\begin{aligned}
f(B)-f(A) & =f^{\prime}(0) \int_{0}^{1}\left(\int_{-1}^{1}(1-\lambda((1-t) A+t B))^{-1}\right. \\
& \left.\times(B-A)(1-\lambda((1-t) A+t B))^{-1} d \mu(\lambda)\right) d t
\end{aligned}
$$

and by Fubini's theorem we get 2.1 .

Corollary 3.1. With the assumptions of Theorem 3, we have the norm inequality

$$
\begin{aligned}
\|f(B)-f(A)\| & \leq f^{\prime}(0)\|B-A\| \\
& \times \int_{-1}^{1}\left(\int_{0}^{1}\left\|(1-\lambda((1-t) A+t B))^{-1}\right\|^{2} d t\right) d \mu(\lambda) .
\end{aligned}
$$

Theorem 4. Let $f$ be a nonconstant operator monotone function on $(-1,1)$ that satisfies the representation (1.2), then

$$
\begin{aligned}
& {[f(B)-f(A)](B-A)} \\
& =f^{\prime}(0) \int_{-1}^{1}\left(\int_{0}^{1}\left[(1-\lambda((1-t) A+t B))^{-1}(B-A)\right]^{2} d t\right) d \mu(\lambda)
\end{aligned}
$$

and

$$
\begin{aligned}
& (B-A)[f(B)-f(A)] \\
& =f^{\prime}(0) \int_{-1}^{1}\left(\int_{0}^{1}\left[(B-A)(1-\lambda((1-t) A+t B))^{-1}\right]^{2} d t\right) d \mu(\lambda)
\end{aligned}
$$

for all $A, B$ with $\operatorname{Sp}(A), \operatorname{Sp}(B) \subset(-1,1)$.

Proof. If we multiply the equality 2.1 at the right with $B-A$ we get

$$
\begin{aligned}
& (f(B)-f(A))(B-A) \\
& =f^{\prime}(0) \int_{-1}^{1}\left(\int_{0}^{1}(1-\lambda((1-t) A+t B))^{-1}\right. \\
& \left.\times(B-A)(1-\lambda((1-t) A+t B))^{-1}(B-A) d t\right) d \mu(\lambda) \\
& =f^{\prime}(0) \int_{-1}^{1}\left(\int_{0}^{1}\left[(1-\lambda((1-t) A+t B))^{-1}(B-A)\right]^{2} d t\right) d \mu(\lambda),
\end{aligned}
$$


which proves 2.6 .

The proof of $(2.7)$ follows in a similar way.

We also have:

Theorem 5. Let $f$ be a nonconstant operator monotone function on $(-1,1)$ that satisfies the representation (1.2), then for $A, B$ with $\operatorname{Sp}(A), \operatorname{Sp}(B) \subset(-1,1)$ and

$$
M \geq B-A \geq m,
$$

for some real numbers $M>m$, we have

$$
\begin{aligned}
& M f^{\prime}(0) \int_{-1}^{1}\left[\int_{0}^{1}(1-\lambda((1-t) A+t B))^{-2} d t\right] d \mu(\lambda) \\
& \geq f(B)-f(A) \\
& \geq m f^{\prime}(0) \int_{-1}^{1}\left[\int_{0}^{1}(1-\lambda((1-t) A+t B))^{-2} d t\right] d \mu(\lambda) .
\end{aligned}
$$

Proof. If $B-A \geq m$, then

$$
\begin{aligned}
& (1-\lambda((1-t) A+t B))^{-1}(B-A)(1-\lambda((1-t) A+t B))^{-1} d t \\
& \geq m(1-\lambda((1-t) A+t B))^{-2}
\end{aligned}
$$

for $t \in[0,1]$ and $\lambda \in[-1,1]$, which implies that

$$
f(B)-f(A) \geq m f^{\prime}(0) \int_{-1}^{1}\left[\int_{0}^{1}(1-\lambda((1-t) A+t B))^{-2} d t\right] d \mu(\lambda) .
$$

If $M \geq B-A$, then in a similar way

$$
M f^{\prime}(0) \int_{-1}^{1}\left[\int_{0}^{1}(1-\lambda((1-t) A+t B))^{-2} d t\right] d \mu(\lambda) \geq f(B)-f(A)
$$

and the theorem is proved.

Corollary 5.1. With the assumptions of Theorem 5 and if $B-A \geq m>0$, then

$$
\begin{aligned}
f(B)-f(A) & \geq m f^{\prime}(0)\left[1+\left(\int_{-1}^{1} \lambda d \mu(\lambda)\right)(A+B)\right. \\
& \left.+\frac{1}{3}\left(\int_{-1}^{1} \lambda^{2} d \mu(\lambda)\right)\left(A^{2}+B^{2}+\frac{A B+B A}{2}\right)\right] \\
& \geq 0 .
\end{aligned}
$$

Proof. Since for $x<1$, then

which implies that

$$
\frac{1}{1-x} \geq 1+x
$$

$$
(1-x)^{-2} \geq(1+x)^{2}=1+2 x+x^{2} \geq 0 .
$$

We then get

$$
(1-\lambda((1-t) A+t B))^{-2}
$$




$$
\begin{aligned}
& \geq 1+2 \lambda((1-t) A+t B)+\lambda^{2}((1-t) A+t B)^{2} \\
& =1+2 \lambda((1-t) A+t B) \\
& +\lambda^{2}\left[(1-t)^{2} A^{2}+t(1-t) A B+t(1-t) B A+t^{2} B^{2}\right] \geq 0
\end{aligned}
$$

for $t \in[0,1], \lambda \in[-1,1]$.

If we take the integral over $t$ in this inequality, then we get

$$
\begin{aligned}
& \int_{0}^{1}(1-\lambda((1-t) A+t B))^{-2} d t \\
& \geq 1+\lambda(A+B)+\frac{1}{3} \lambda^{2}\left(A^{2}+B^{2}+\frac{A B+B A}{2}\right) \geq 0
\end{aligned}
$$

for $\lambda \in[-1,1]$.

Taking the integral over $\mu(\lambda)$ we get

$$
\begin{aligned}
& \int_{-1}^{1}\left[\int_{0}^{1}(1-\lambda((1-t) A+t B))^{-2} d t\right] d \mu(\lambda) \\
& \geq 1+\left(\int_{-1}^{1} \lambda d \mu(\lambda)\right)(A+B) \\
& +\frac{1}{3}\left(\int_{-1}^{1} \lambda^{2} d \mu(\lambda)\right)\left(A^{2}+B^{2}+\frac{A B+B A}{2}\right) \geq 0,
\end{aligned}
$$

which, by the second inequality in $(2.9)$ gives the desired result 2.10 .

In the following, in order to simplify terminology, when we write $T \geq 0$ we automatically assume that the operator $T$ is selfadjoint.

We give now some necessary and sufficient conditions for the operators $A, B$ with $\operatorname{Sp}(A), \operatorname{Sp}(B) \subset(-1,1)$ such that the inequality $(B-A)(f(B)-f(A)) \geq$ 0 holds.

Theorem 6. Let $A, B$ with $\operatorname{Sp}(A), \operatorname{Sp}(B) \subset(-1,1)$. The following statements are equivalent:

(i) For all for $\lambda \in[-1,1]$,

$$
(1-\lambda B)^{-1}(1-\lambda A)+(1-\lambda A)^{-1}(1-\lambda B) \geq 2 .
$$

(ii) For all for $\lambda \in[-1,1]$,

$$
\int_{0}^{1}\left[(1-\lambda[(1-t) B+\lambda t A])^{-1}(B-A)\right]^{2} d t \geq 0 .
$$

(iii) For all $\lambda \in[-1,1]$

$$
\left(\kappa_{\lambda}(B)-\kappa_{\lambda}(A)\right)(B-A) \geq 0,
$$

where $\kappa_{\lambda}(t)=t(1-\lambda t)^{-1}, t \in[-1,1]$.

(iv) For all operator monotone function $f$ on $(-1,1)$,

$$
(f(B)-f(A))(B-A) \geq 0 .
$$


(v) For all operator monotone function $f$ on $(-1,1)$,

$$
(B-A)(f(B)-f(A)) \geq 0 .
$$

Proof. Consider the continuous function $f$ defined on an interval $I$ for which the corresponding operator function is Gâteaux differentiable and for $C, D$ selfadjoint operators with spectra in $I$ we consider the auxiliary function defined on $[0,1]$ by

$$
f_{C, D}(t)=f((1-t) C+t D), t \in[0,1] .
$$

If $f_{C, D}$ is Gâteaux differentiable on the segment $[C, D]:=\{(1-t) C+t D, t \in[0,1]\}$, then we have, by the properties of the Bochner integral, that

$$
f(D)-f(C)=\int_{0}^{1} \frac{d}{d t}\left(f_{C, D}(t)\right) d t=\int_{0}^{1} \nabla f_{(1-t) C+t D}(D-C) d t .
$$

If we write this equality for the function $f(t)=-t^{-1}$ and $C, D>0$, then we get the representation

$$
C^{-1}-D^{-1}=\int_{0}^{1}((1-t) C+t D)^{-1}(D-C)((1-t) C+t D)^{-1} d t
$$

From (2.14) we have

$$
\begin{aligned}
& (1-\lambda B)^{-1}-(1-\lambda A)^{-1} \\
& =\int_{0}^{1}((1-t)(1-\lambda B)+t(1-\lambda A))^{-1}((1-\lambda A)-(1-\lambda B)) \\
& \times((1-t)(1-\lambda B)+t(1-\lambda A))^{-1} d t \\
& =\lambda \int_{0}^{1}(1-\lambda[(1-t) B+\lambda t A])^{-1}(B-A) \\
& \times(1-\lambda[(1-t) B+\lambda t A])^{-1} d t
\end{aligned}
$$

for $\lambda \in[-1,1]$.

If we multiply this equality at right by $B-A$, we get

$$
\begin{aligned}
& {\left[(1-\lambda B)^{-1}-(1-\lambda A)^{-1}\right](B-A)} \\
& =\lambda \int_{0}^{1}(1-\lambda[(1-t) B+\lambda t A])^{-1}(B-A) \\
& \times(1-\lambda[(1-t) B+\lambda t A])^{-1}(B-A) d t \\
& =\lambda \int_{0}^{1}\left[(1-\lambda[(1-t) B+\lambda t A])^{-1}(B-A)\right]^{2} d t
\end{aligned}
$$

for $\lambda \in[-1,1], \lambda \neq 0$, namely

$$
\begin{aligned}
V_{\lambda} & :=\frac{1}{\lambda}\left[(1-\lambda B)^{-1}-(1-\lambda A)^{-1}\right](B-A) \\
& =\int_{0}^{1}\left[(1-\lambda[(1-t) B+\lambda t A])^{-1}(B-A)\right]^{2} d t .
\end{aligned}
$$


Also,

$$
\begin{aligned}
& {\left[(1-\lambda B)^{-1}-(1-\lambda A)^{-1}\right](B-A)} \\
& =\frac{1}{\lambda}\left[(1-\lambda B)^{-1}-(1-\lambda A)^{-1}\right](\lambda B-\lambda A) \\
& =\frac{1}{\lambda}\left[(1-\lambda B)^{-1}-(1-\lambda A)^{-1}\right](1-\lambda A-(1-\lambda B)) \\
& =\frac{1}{\lambda}\left[(1-\lambda B)^{-1}(1-\lambda A)+(1-\lambda A)^{-1}(1-\lambda B)-2\right],
\end{aligned}
$$

which gives that

$$
\begin{aligned}
V_{\lambda} & =\frac{1}{\lambda}\left[(1-\lambda B)^{-1}-(1-\lambda A)^{-1}\right](B-A) \\
& =\frac{1}{\lambda^{2}}\left[(1-\lambda B)^{-1}(1-\lambda A)+(1-\lambda A)^{-1}(1-\lambda B)-2\right]
\end{aligned}
$$

for $\lambda \in[-1,1], \lambda \neq 0$.

We conclude that

$$
\begin{aligned}
& \left(\kappa_{\lambda}(B)-\kappa_{\lambda}(A)\right)(B-A) \\
& =\frac{1}{\lambda^{2}}\left[(1-\lambda B)^{-1}(1-\lambda A)+(1-\lambda A)^{-1}(1-\lambda B)-2\right] \\
& =\int_{0}^{1}\left[(1-\lambda[(1-t) B+\lambda t A])^{-1}(B-A)\right]^{2} d t
\end{aligned}
$$

for $\lambda \in[-1,1], \lambda \neq 0$.

We observe that the equivalence between the statements (i), (ii) and (iii) follows directly from the identity (2.15).

Since the function $\kappa_{\lambda}(t)=t(1-\lambda t)^{-1}$ is operator monotone on $(-1,1)$, the statement (iv) implies (iii).

If (ii) holds, then by representation (2.6) we have

$$
\begin{aligned}
& {[f(B)-f(A)](B-A)} \\
& =f^{\prime}(0) \int_{-1}^{1}\left(\int_{0}^{1}\left[(1-\lambda((1-t) A+t B))^{-1}(B-A)\right]^{2} d t\right) d \mu(\lambda) \\
& \geq 0,
\end{aligned}
$$

which shows that (iv) holds.

Define the operator $K:=(f(B)-f(A))(B-A)$. Since

$$
\begin{aligned}
K^{*} & =[(f(B)-f(A))(B-A)]^{*}=(B-A)^{*}(f(B)-f(A))^{*} \\
& =(B-A)(f(B)-f(A))
\end{aligned}
$$

then the fact that $K$ is selfadjoint is equivalent to

$$
(f(B)-f(A))(B-A)=(B-A)(f(B)-f(A)),
$$

which is also equivalent to the fact that

$$
f(A) B+f(B) A=B f(A)+A f(B) .
$$


These prove the equivalence between (iv) and (v).

Therefore, we can state:

Corollary 6.1. Let $A, B$ with $\operatorname{Sp}(A), \operatorname{Sp}(B) \subset(-1,1)$. The statement (i) is equivalent to the inequality

$$
f(B) B+f(A) A \geq f(A) B+f(B) A,
$$

for all $f$ an operator monotone function on $(-1,1)$.

In the note 4 Fujii and Nakamoto showed that the inequality (2.13) does not hold in general for $A, B$ with $\operatorname{Sp}(A), \operatorname{Sp}(B) \subset(-1,1)$. They also proved that if $C, D>0$ and $C D^{-1}+D C^{-1}$ is selfadjoint, then

$$
C D^{-1}+D C^{-1} \geq 2 \text {. }
$$

Indeed, as shown in [4, if we put $T=C D^{-1}$, then $V=T+T^{-1}$ is selfadjoint by the assumption. Note that the spectrum $\operatorname{Sp}(T)$ of $T$ is included in $(0, \infty)$, because $C, D>0$ and $\operatorname{Sp}(T)=\operatorname{Sp}\left(C^{1 / 2} D^{-1} C^{1 / 2}\right)$. Since $\operatorname{Sp}(V)=\left\{t+\frac{1}{t}, t \in \operatorname{Sp}(T)\right\}$ by the spectral mapping theorem for rational functions, hence we have $T+T^{-1} \geq 2$.

Observe that, in fact we have:

Proposition 2.1. Let $A, B$ with $\operatorname{Sp}(A), \operatorname{Sp}(B) \subset(-1,1)$, then the statements (i) and

(i') The operator $A(1-\lambda B)^{-1}+B(1-\lambda A)^{-1}$ is selfadjoint for all $\lambda \in[-1,1]$, are equivalent.

Proof. Notice that for all $\lambda \in[-1,1]$,

$$
\begin{aligned}
& (1-\lambda B)^{-1}(1-\lambda A)+(1-\lambda A)^{-1}(1-\lambda B) \\
& =(1-\lambda B)^{-1}+(1-\lambda A)^{-1}-\lambda\left[(1-\lambda B)^{-1} A+(1-\lambda A)^{-1} B\right] .
\end{aligned}
$$

Also, the operator $(1-\lambda B)^{-1}+(1-\lambda A)^{-1}$ is selfadjoint for $\lambda \in[-1,1]$.

If the statement $(i)$ holds, then $(1-\lambda B)^{-1}(1-\lambda A)+(1-\lambda A)^{-1}(1-\lambda B)$ is selfadjoint and by 2.18 we must have that $(1-\lambda B)^{-1} A+(1-\lambda A)^{-1} B$ is selfadjoint, which shows that

$$
\left((1-\lambda B)^{-1} A+(1-\lambda A)^{-1} B\right)^{*}=A(1-\lambda B)^{-1}+B(1-\lambda A)^{-1}
$$

is selfadjoint, namely $\left(i^{\prime}\right)$ is true.

If the statement $\left(i^{\prime}\right)$ holds, then by 2.18 we get

$$
(1-\lambda B)^{-1}(1-\lambda A)+(1-\lambda A)^{-1}(1-\lambda B)
$$

is selfadjoint and by 2.17 for $C=(1-\lambda B)^{-1}, D=(1-\lambda A)^{-1}$ we obtain the inequality (2.11), namely (i) is true.

We define the class of operators

$\mathfrak{C l}_{(-1,1)}(H):=\left\{(A, B) \mid \operatorname{Sp}(A), \operatorname{Sp}(B) \subset(-1,1)\right.$ and satisfy condition $\left.\left(i^{\prime}\right)\right\}$.

We observe that if $(A, B) \in \mathfrak{C l}_{(-1,1)}(H)$ then $(B, A) \in \mathfrak{C l}_{(-1,1)}(H)$. 
Also if $A B=B A$ with $\operatorname{Sp}(A), \operatorname{Sp}(B) \subset(-1,1)$ then $V_{\lambda}:=(1-\lambda B)^{-1}(1-\lambda A)$ and $V_{\lambda}^{-1}=(1-\lambda A)^{-1}(1-\lambda B), \lambda \in[-1,1]$, are selfadjoint and since $V_{\lambda}+V_{\lambda}^{-1} \geq$ $2, \lambda \in[-1,1]$ we derive that $(A, B) \in \mathfrak{C l}_{(-1,1)}(H)$. Therefore, if $\mathfrak{C o}_{(-1,1)}(H)$ is the class of all pairs of commutative operators $A, B$ with $\operatorname{Sp}(A), \operatorname{Sp}(B) \subset(-1,1)$, then we have

$$
\emptyset \neq \mathfrak{C o}_{(-1,1)}(H) \subset \mathfrak{C l}_{(-1,1)}(H)
$$

\section{Discrete Inequalities}

We have the following Chebychev type operator inequality:

Proposition 3.1. Assume that the function $f:(-1,1) \rightarrow \mathbb{R}$ is operator monotone in $(-1,1)$. If $g: I \rightarrow(-1,1)$ is continuous, then for all selfadjoint operators $A_{k}$, $k=1, \ldots, n$ with spectra in I such that $\left(g\left(A_{k}\right), g\left(A_{j}\right)\right) \in \mathfrak{C l}_{(-1,1)}(H)$ for all $k$, $j=1, \ldots, n$ and $p_{k} \geq 0, k=1, \ldots, n$ with $\sum_{k=1}^{n} p_{k}=1$, we have

$$
\sum_{k=1}^{n} p_{k}(f \circ g)\left(A_{k}\right) g\left(A_{k}\right) \geq \sum_{k=1}^{n} p_{k} g\left(A_{k}\right) \sum_{k=1}^{n} p_{k}(f \circ g)\left(A_{k}\right) .
$$

If $A_{k}, k=1, \ldots, n$, with $\operatorname{Sp}\left(A_{k}\right) \subset(-1,1),\left(A_{k}, A_{j}\right) \in \mathfrak{C l}_{(-1,1)}(H)$ for all $k$, $j=1, \ldots, n$ and $p_{k} \geq 0, k=1, \ldots, n$ with $\sum_{k=1}^{n} p_{k}=1$, then

$$
\sum_{k=1}^{n} p_{k} f\left(A_{k}\right) A_{k} \geq \sum_{k=1}^{n} p_{k} A_{k} \sum_{k=1}^{n} p_{k} f\left(A_{k}\right)
$$

Proof. From 2.16) we get

$$
\begin{aligned}
& (f \circ g)\left(A_{k}\right) g\left(A_{k}\right)+(f \circ g)\left(A_{j}\right) g\left(A_{j}\right) \\
& \geq(f \circ g)\left(A_{j}\right) g\left(A_{k}\right)+(f \circ g)\left(A_{k}\right) g\left(A_{j}\right)
\end{aligned}
$$

for all $k, j \in\{1, \ldots, n\}$.

If we multiply (3.3) by $p_{k} p_{j} \geq 0$ and sum over $k$ and $j$ from 1 to $n$, we get

$$
\begin{aligned}
& \sum_{k=1}^{n} \sum_{j=1}^{n} p_{k} p_{j}(f \circ g)\left(A_{k}\right) g\left(A_{k}\right)+\sum_{k=1}^{n} \sum_{j=1}^{n} p_{k} p_{j}(f \circ g)\left(A_{j}\right) g\left(A_{j}\right) \\
& \geq \sum_{k=1}^{n} \sum_{j=1}^{n} p_{k} p_{j}(f \circ g)\left(A_{j}\right) g\left(A_{k}\right)+\sum_{k=1}^{n} \sum_{j=1}^{n} p_{k} p_{j}(f \circ g)\left(A_{k}\right) g\left(A_{j}\right),
\end{aligned}
$$

which is equivalent to

$$
\begin{aligned}
& \sum_{k=1}^{n} p_{k}(f \circ g)\left(A_{k}\right) g\left(A_{k}\right)+\sum_{j=1}^{n} p_{j}(f \circ g)\left(A_{j}\right) g\left(A_{j}\right) \\
& \geq \sum_{j=1}^{n} p_{j}(f \circ g)\left(A_{j}\right) \sum_{k=1}^{n} p_{k} g\left(A_{k}\right)+\sum_{k=1}^{n} p_{k}(f \circ g)\left(A_{k}\right) \sum_{j=1}^{n} p_{j} g\left(A_{j}\right)
\end{aligned}
$$

that is equivalent to the first part of (3.1). 
Remark 3.1: If $A_{k}, k=1, \ldots, n$, with $\operatorname{Sp}\left(A_{k}\right) \subset(-1,1),\left(A_{k}, A_{j}\right) \in \mathfrak{C l}_{(-1,1)}(H)$ for all $k, j=1, \ldots, n$ and $p_{k} \geq 0, k=1, \ldots, n$ with $\sum_{k=1}^{n} p_{k}=1$, then by $(3.2)$,

$$
\sum_{k=1}^{n} p_{k} A_{k} \tan \left(A_{k}\right) \geq \sum_{k=1}^{n} p_{k} A_{k} \sum_{k=1}^{n} p_{k} \tan \left(A_{k}\right) \text {. }
$$

Also for $r \in(0,1)$,

$$
\begin{aligned}
& \sum_{k=1}^{n} p_{k} A_{k}\left[\left(1_{H}+A_{k}\right)\left(1_{H}-A_{k}\right)^{-1}\right]^{r} \\
& \geq \sum_{k=1}^{n} p_{k} A_{k} \sum_{k=1}^{n} p_{k}\left[\left(1_{H}+A_{k}\right)\left(1_{H}-A_{k}\right)^{-1}\right]^{r} .
\end{aligned}
$$

We also have

$$
\begin{aligned}
& \sum_{k=1}^{n} p_{k} A_{k} \ln \left[\left(1_{H}+A_{k}\right)\left(1_{H}-A_{k}\right)^{-1}\right] \\
& \geq \sum_{k=1}^{n} p_{k} A_{k} \sum_{k=1}^{n} p_{k} \ln \left[\left(1_{H}+A_{k}\right)\left(1_{H}-A_{k}\right)^{-1}\right] .
\end{aligned}
$$

Acknowledgement. The author would like to thank Professors M. Fujii and R. Nakamoto for the private note [4].

\section{REFERENCES}

[1] M. Bagher Ghaemi, V. Kaleibary, Some inequalities involving operator monotone functions and operator means. Math. Inequal. Appl.19 (2016), no. 2, 757-764.

[2] R. Bhatia, Matrix Analysis. Graduate Texts in Mathematics, 169. Springer-Verlag, New York, 1997. xii +347 pp. ISBN: 0-387-94846-5.

[3] G. L. Brown and M. Uchiyama, Some results on strongly operator convex functions and operator monotone functions. Linear Algebra Appl. 553 (2018), 238-251.

[4] M. Fujii and R. Nakamoto, Note on Dragomir's theorems, Private note, 5 Aug. 2020. Preprint RGMIA Res. Rep. Coll. 23 (2020), Art. 113, 4 pp.

[5] J. I. Fujii, Y. Seo, On parametrized operator means dominated by power ones, Sci. Math. 1 (1998) 301-306.

[6] T. Furuta, Concrete examples of operator monotone functions obtained by an elementary method without appealing to Löwner integral representation, Linear Algebra and its Applications 429 (2008) 972-980.

[7] T. Furuta, Precise lower bound of $f(A)-f(B)$ for $A>B>0$ and non-constant operator monotone function $f$ on $[0, \infty)$. J. Math. Inequal. 9 (2015), no. 1, 47-52.

[8] E. Heinz, Beiträge zur Störungsteorie der Spektralzerlegung, Math. Ann. 123 (1951) 415438.

[9] H. Jafarmanesh, M. Khosravi and A. Sheikhhosseini, Some operator inequalities involving operator monotone functions. Bull. Sci. Math. 166 (2021), Paper No. 102938, 10 pp.

[10] K. Löwner, Über monotone MatrixFunktionen, Math. Z. 38 (1934) 177-216.

[11] F. Kubo, T. Ando, Means of positive linear operators, Math. Ann. 246 (1980) 205-224.

[12] J. Mićić, H. R. Moradi, Some inequalities involving operator means and monotone convex functions. J. Math. Inequal. 14 (2020), no. 1, 135-145. 
[13] M. Uchiyama, Operator monotone functions, Jacobi operators and orthogonal polynomials, J. Math. Anal. Appl. 401 (2013) 501-509.

[14] H. Zuo, G. Duan, Some inequalities of operator monotone functions. J. Math. Inequal. 8 (2014), no. 4, 777-781.

1 Mathematics, College of Engineering \& Science

Victoria University, PO Box 14428

Melbourne City, MC 8001, Australia.

Email address: sever.dragomir@vu.edu.au

$U R L:$ http://rgmia.org/dragomir

2 DSt-nRF Centre of Excellence in the Mathematical and Statistical Sciences, School of Computer Science \& Applied Mathematics,

UNiversity OF THE WitwatersRand,

Johannesburg, South Africa.

Received: 28.4.2021

Revised: 5.9 .2021

Accepted: 6.9.2021 